\title{
El Observatorio Astronómico de Honduras: Un Proyecto de Cooperación Internacional
}

\author{
María Cristina Pineda de Carías \\ Observatorio Astronómico Centroamericano de Suyapa \\ Universidad Nacional Autónoma de Honduras \\ Ciudad Universitaria, Tegucigalpa M. D. C., Honduras \\ Email: mcarias@cablecolor.hn
}

\begin{abstract}
RESUMEN
Se describe la historia, la situación actual y los logros en Astronomía y Astrofísica como campos académicos de la Universidad Nacional Autónoma de Honduras. La primera actividad fue el Proyecto "Un Observatorio Astronómico para Centro América: una manera realista de fortalecer la ciencia espacial básica en países en desarrollo", porque es el marco para incorporar todo el trabajo inicial para el desarrollo de las diferentes actividades académicas, sobre la base del cual el Observatorio Astronómico Centroamericano de Suyapa fue sacado adelante como un proyecto de permanente cooperación internacional. La docencia, investigación y actividades de extensión asociada se describen para ilustrar el modelo bien organizado de esta unidad académica con reconocimiento internacional. Finalmente se comenta sobre el alcance regional e internacional de este Proyecto de Astronomía Centroamericana en Honduras.
\end{abstract}

Palabras clave: Observatorio Astronómico Honduras, docencia, investigación, extensión.

\begin{abstract}
The history, current situation and achievements in Astronomy and Astrophysics as academicals fields of the National Autonomous University of Honduras are described. The first activity was the Project "An Astronomical Observatory for Central America: a realistic way of strengthening basic space science in developing countries", because it is the frame incorporating all the initial work for the development of the different academic activities, on the basis of which the Central America Suyapa Astronomical Observatory was brought forward in a project of permanent international cooperation. The associated education, research and outreach activities are described to illustrate the well organized model of this academic unit with international recognition. Finally we comment on the regional and international scope of this Central America Astronomy Project in Honduras.
\end{abstract}

Key words: Astronomical Observatory of Honduras, education, research, outreach. 


\section{Introducción}

En Centroamérica, la iniciativa para crear el primer observatorio astronómico de los tiempos modernos surgió en Honduras a inicio de la última década del siglo veinte. El Proyecto, "Un Observatorio Astronómico para Centro América: Una manera realista de fortalecer la ciencia espacial básica en los países en desarrollo” (Pineda de Carías, 1995) contiene las bases para el establecimiento de un centro astronómico regional. Para el desarrollo de este proyecto regional, una estrategia basada en la permanente cooperación internacional ha sido organizada alrededor de las siguientes actividades principales:

- Asamblea de Astrónomos de América Central;

- Cursos Centroamericanos de Astronomía y Astrofísica;

- Programa de Maestría Centroamericana en Astronomía y Astrofísica;

- Facilidades observacionales centroamericanas; y,

- Acuerdos de Cooperación Internacional.

En julio de 1994, las autoridades de la Universidad Nacional Autónoma de Honduras (UNAH) aprobaron el establecimiento del Observatorio Astronómico de la Universidad (OA/UNAH) como una unidad académica responsable de:

1) Desarrollar observaciones y proyectos de investigación científica en el campo de la astronomía y otras áreas afines;

2) Manejar y desarrollar facilidades instrumentales para las observaciones, reducción y análisis, y para recibir imágenes astronómicas:

3) Proporcionar servicios básicos a los estudiantes de diferentes niveles del Sistema Educativo Nacional y para el público en general;

4) Organizar y coordinar actividades de extensión y Programas de Educación en Astronomía para la diseminación del conocimiento astronómico.

En junio de 1997, dentro del marco del VII Taller de las Naciones Unidas y la Agencia Europea Espacial sobre Ciencia Espacial Básica realizado en Tegucigalpa, Honduras (véase el Informe del VII UN/ESA Workshop, 1997) y con la participación de 80 científicos de unas 30 Agencias y Organizaciones, el Observatorio OA/UNAH se renombró para el más amplio e internacional "Observatorio Astronómico Centroamericano de Suyapa (OACS)", abriendo una nueva Era de desarrollo y establecimiento de la Astronomía y la Astrofísica en Centro América. El primer acto fue la inauguración de la primera facilidad observacional regional, el Telescopio "René Sagastume Castillo", un telescopio óptico Schmidt Cassegrain de 42 centímetros, inmediatamente seguido de la apertura del Programa de Maestría en Astronomía y Astrofísica, un programa de postgrado para ingenieros, físicos y matemáticos que, después de haber completado una carrera universitaria de 5 años, querían continuar con la Astronomía como una carrera profesional en Centro América.

En el presente, el OACS/UNAH es el único centro de observación e investigación en una universidad centroamericana. El nivel de organización que ha alcanzado ahora puede ser considerado como un modelo para el desarrollo académico para el establecimiento de la 
Ciencia Espacial, por medio de la docencia, la investigación, la extensión y la administración. El trabajo hecho en el OACS/UNAH es muy importante y altamente reconocido. Ha contribuido a:

- Dar presencia a Honduras y los países de Centro América en el desarrollo de la ciencia y las tecnologías espaciales, incluyendo la Astronomía y la Astrofísica;

- Incrementar los índices de recursos humanos en Centro América formados al más alto nivel;

- Incrementar la investigación y la producción tecnológica en Centro América;

- Abrir nuevos campos y crear nuevas oportunidades de formación para las nuevas generaciones;

- Fortalecer, en general, lazos de cooperación internacional entre universidades, y en particular entre los observatorios astronómicos y centros de investigación.

Está aun en agenda insertar a Centro América en proyectos de investigación y exploración espacial. Sin embargo, se debe reconocer que algunos pasos ya se han dado en el nivel universitario (OACS/UNAH), con el objeto de continuar con las políticas nacionales de ciencia y tecnología que miran a proyectos mundiales tales como el Observatorio Espacial del Mundo (Wamsteker and Shustov, 2003).

\section{El Observatorio Astronómico de Honduras}

El Observatorio Astronómico Centroamericano de Suyapa de Honduras (ver Figura 1) está localizado en la Ciudad Universitaria de la Universidad Nacional Autónoma de Honduras (Latitud: $14^{\circ} 05^{\prime} \mathrm{N} ; 87^{\circ} 09^{\prime} \mathrm{W}$; Altura: 1076 metros sobre el nivel del mar). El sitio del observatorio fue escogido dentro del campus universitario, con el objeto de proporcionar facilidades a los estudiantes para hacer sus propias observaciones y también para proporcionar acceso público al observatorio de manera que los ciudadanos puedan aprender acerca de la Astronomía.

El telescopio principal del OACS/UNAH es un LX200 Schmidt Cassegrain con una montura ecuatorial (Latitud: $14^{\circ}$ Norte). El tamaño del espejo primario es de 42 centímetros y la razón focal es de f/10 ó f/6. El telescopio está equipado con una cámara CCD y una rueda de filtros. Revistas científicas, libros de texto, catálogos astronómicos y software especializado (es decir, IRAF sobre una plataforma Linux) están disponibles para la reducción y el análisis de los datos de imágenes astronómicas obtenidas en el OACS/UNAH o en cualquier otro centro de observación, apoyado en conexiones permanentes a Internet (24 horas al día, 7 días a la semana). 


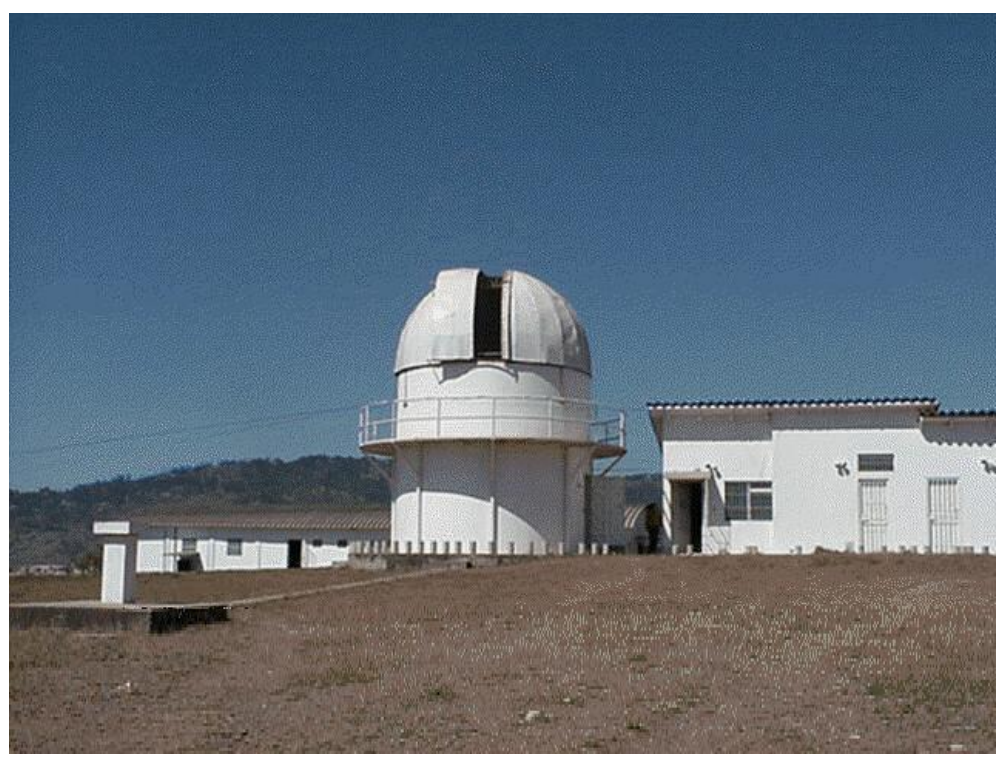

Figura 1. El Observatorio Astronómico Centroamericano de Suyapa, inaugurado el 18 de junio de 1997 en el VII Taller de las Naciones Unidas y la Agencia Europea Espacial sobre Ciencia Espacial Básica en Tegucigalpa, Honduras. En esta figura se puede ver la cúpula que alberga el telescopio óptico de 42 centímetros, y los edificios de las oficinas y salones de clase.

Las principales actividades de trabajo del OACS/UNAH son: docencia, investigación y extensión.

a) Docencia. En el campo de la Educación en Astronomía, el OACS/UNAH ofrece y activamente apoya lo siguiente:

- Maestría en Astronomía y Astrofísica. Un programa de postgrado regional dirigido a la formación de profesionales con la responsabilidad de establecer y desarrollar la Astronomía y la Astrofísica en la región, por medio de la cooperación internacional. Desde 1998, cuando el programa fue abierto, la colaboración de astrónomos provenientes de universidades y observatorios astronómicos de Argentina, Brasil, Francia, España, Estados Unidos y México han estado disponibles; con algunas instituciones habiendo firmado Convenios Internacionales de Cooperación. En visitas de uno o dos meses, estos astrónomos vienen al OACS/UNAH como profesores para enseñar diferentes cursos en sus propios campos de interés, y también como tutores de los proyectos de tesis. Hasta ahora, tres promociones de estudiantes, la MAA-98, la MAA-99 y la MAA.2001, cada dos años han tomado las 20 asignaturas que comprende el Plan de Estudios de la Maestría, y han iniciado sus proyectos de investigación, con algunos de ellos habiendo tenido la oportunidad de viajar a Argentina, desde uno hasta seis meses, a las instituciones extranjeras donde sus tutores trabajan. En el 2002, los primeros estudiantes de postgrado terminaron su trabajo, y desde aquí en adelante, más estudiantes están terminando su carrera de Máster en Astronomía y Astrofísica.

- AN111 Introducción a la Astronomía (4 unidades valorativas). Es una asignatura general y optativa para los estudiantes de todas las carreras de la 
Universidad. Observaciones y modelos, Sistema Solar, Estrellas y Medio Interestelar, y Galaxias y Cosmología, son las cuatro unidades del programa académico desarrollado en un curso de un semestre, con clases teóricas complementadas con algunas actividades prácticas y observacionales. Cada año, 5 miembros del cuerpo de profesores del OACS/UNAH enseña a unos 500 estudiantes universitarios de unas 30 carreras.

- Cursos Centroamericanos de Astronomía y Astrofísica (CURCAA). Estos son cursos regionales donde los participantes tienen la oportunidad de discutir tópicos relevantes sobre el Sol, Sistema Solar, formación y evolución estelar, medio interestelar, galaxias y cosmología, y técnicas de observación y reducción de datos. Estos cursos también promueven el intercambio de ideas y experiencias entre docentes y estudiantes trabajando en Astronomía y Astrofísica. Entre 1995 y 2001, seis CURCAA han sido desarrollados, cada uno en los países centroamericanos de Honduras (1995), El Salvador (1996), Guatemala (1997), Panamá (1998), Nicaragua (1999) y Costa Rica (2001). En un segundo ciclo, el CURCAA regresó a Honduras en 2002, para continuar en El Salvador en 20003.

Estos CURCAA son programados dentro del marco de la Asamblea de Astrónomos de América Central (AAAC), un organismo regional ya reconocido por la Unión Astronómica Internacional (IAU) para promover el desarrollo de la Astronomía por medio de la cooperación internacional permanente. La AAAC tiene sus propias leyes y reglamentos, y sesiones son programadas en fechas periódicas, teniendo una Junta Directiva que se cambia cada dos años.

b) Investigación. Como un resultado del nivel alcanzado en el OACS/UNAH, varias áreas de investigación han sido identificadas y empiezan a producir algunos resultados, en una escala nacional e internacional:

- Educación en Astronomía. Empezando con el Proyecto "Un Observatorio Astronómico para Centro América: una manera realista para fortalecer la ciencia espacial básica en países en desarrollo" (Pineda de Carías, 1995), varios documentos en Educación en Astronomía han sido publicados, entre los cuales especial atención merece "El Programa de Maestría Centroamericano en Astronomía y Astrofísica" (Pineda de Carías, 2001), el primer documento presentado en una Asamblea General de la Unión Astronómica Internacional, por un astrónomo centroamericano.

- Observaciones Astronómicas en el OACS/UNAH (I). Un proyecto que estudia las condiciones de observación del sitio del OACS, y el tipo de programas de observación que pueden ser desarrollados con el telescopio LX200 "René Sagastume Castillo". Siguiendo el modelo diseñado para este proyecto, cuatro líneas son exploradas: i) El sitio, con el objeto de caracterizar el sitio donde el telescopio ha sido colocado en el campus universitario; ii) El telescopio y sus accesorios, con el objeto de determinar el funcionamiento de los instrumentos y las facilidades ya instaladas; iii) Recursos Humanos, para encontrar quiénes son los usuarios y en qué proyectos de observación ellos están interesados; y iv) Observaciones Astronómicas, para desarrollar diferentes programas de observación para 
encontrar cual se ajusta mejor a las condiciones del sitio, los instrumentos ya instalados y los recursos humanos involucrados.

- Observaciones Astronómicas en el OACS/UNAH (II). Este es otro proyecto que busca estudiar aquellos eventos astronómicos tales como eclipses, lluvias de meteoros o cometas, visibles desde el territorio Hondureño, antes, durante y después de su ocurrencia o aparecimiento. Algunos artículos ya publicados en estos campos son: "Acerca de algunas mediciones hechas en Honduras durante el eclipse solar total del 11 de julio de 1991" (AIP, 1993), y "Acerca de una gran bola de fuego vista en Honduras" (Meteoroids, 1998).

- Dinámica de Sistemas Planetarios. Evolución de diferentes objetos de nuestro Sistema Solar y de otros sistemas planetarios alrededor de otras estrellas es estudiado. Actualmente, hay dos áreas en desarrollo: 1) Objetos menores (asteroides) del Sistema Solar, un área que después de la tesis de Maestría en Astronomía y Astrofísica: "Acerca de las perturbaciones seculares en la zona exterior de Saturno" ha derivado en el estudio de Inestabilidades en la Zona Exterior de Saturno y Asteroides Binarios. 2) Planetas extrasolares, actualmente estudiando la posibilidad de existencia de planetas terrestres.

- Atmósferas estelares. La estructura dinámica y termodinámica de material circumestelar en sistemas binarios de corto período es estudiada. Después de haber buscado datos adecuados de binarias en el UV (INES / IUE, Wamsteker et al., 2000), para la identificación de líneas espectrales específicas, la aplicación de metodologías especiales, y después de haber completado campañas observacionales, un estudio de la dinámica y la termodinámica del material circumestelar en binarias interactuantes esta en desarrollo.

- Actividad Solar. Una relación entre la intensidad del cielo de fondo, eyecciones de masa coronal y los efectos geomagnéticos derivados es estudiado. A través de investigación bibliográfica acerca de coronografía, observaciones coronográficas, eyecciones de masa coronal y otros fenómenos dinámicos en la atmósfera solar y fenómenos geomagnéticas; y a través de análisis estadísticos que correlacionan la dinámica solar y fenómenos geomagnéticos, derivados de datos obtenidos del MICA (Mirror Coronograph for Argentina); una relación entre la intensidad del cielo de fondo como indicador de eyecciones de masa coronal es estudiada.

- Arqueoastronomía Maya. Evidencia de actividad astronómica entre los Mayas, por medio del estudio de las fechas y orientación de las estructuras y monumentos de Copán, Honduras, es estudiado. En este proyecto, astrónomos y arqueólogos están trabajando juntos en el OACS/UNAH, estudiando planos, mapas, documentos bibliográficos del sitio arqueológico de Copán; después de escoger el área y el objeto astronómico de estudio, el problema es establecido y la metodología de investigación; estructuras y monumentos específicos son documentados, y observaciones astronómicas son hechas con el objeto de obtener resultados. Actualmente hay dos áreas en desarrollo: alineamientos y orientaciones de estructuras y monumentos, y ciclos del calendario. 
- Percepción Remota. Después de los daños del Huracán Mitch en Honduras la necesidad de monitoreo permanente del territorio y la necesidad de evaluación del impacto del daño usando técnicas espaciales fue develada. Después que un Laboratorio de Percepción Remota fue organizado y abierto en el OACS/UNAH. Parte importante del Laboratorio de Percepción Remota es el Sistema de Información Geográfica ya conformado con imágenes de satélite y fotografías aéreas de Honduras y Centro América, para diferentes años. Un Proyecto de la NASA/CCAD del Corredor Biológico Mesoamericano, Proyectos de Clasificación de la cobertura y el uso de la tierra, y la Caracterización y detección del cambio de áreas protegidas, son algunos de los proyectos que están en desarrollo.

c) Extensión. Con el objeto de contribuir a divulgar y diseminar el conocimiento astronómico, los siguientes proyectos de extensión están siendo desarrollados en el OACS/UNAH:

- Efemérides Astronómicas. Las Efemérides del Sol, la Luna, los planetas y eventos especiales de Honduras y Centro América son preparadas y divulgadas como una publicación mensual. Ediciones especiales de estas efemérides son preparadas para aquellos eventos que llaman la atención del público en general tales como eclipses, lluvias de meteoros, pasos del Sol por el cenit, equinoccios, solsticios y otros.

- Visitas Académicas al OACS/UNAH. Bajo el lema "Desde Honduras, Centro América: una ventana hacia el Universo", un programa de visitas educativas y para personas interesadas se desarrolla actualmente tres días a la semana. Estudiantes y profesores de escuelas primarias, colegios de secundaria y universitarios, participan de conferencias, exhibiciones, actividades prácticas y observaciones astronómicas. Cada año, unos 4000 estudiantes de diferentes niveles del sistema educativo nacional disfrutan y se benefician de este importante proyecto.

- Noches Astronómicas. Cada viernes por la noche, por unas dos horas, niños, jóvenes y personas adultas visitan el OACS/UNAH para escuchar charlas especiales dirigidas a popularizar la Astronomía en todos los niveles, y también permitir a la gente hacer observaciones astronómicas de la luna, los planetas, objetos del cielo profundo y eventos astronómicos especiales. Cada año el OACS/UNAH recibe unas 5,000 personas de todas las edades, y este programa es bien conocido y altamente reconocido dentro del país.

\section{Algunos comentarios finales}

Como comentarios finales los siguientes deben ser enfatizados:

- Con respecto a hacer investigación y la formación de astrónomos para Centro América, estos son actualmente los principales objetivos detrás del establecimiento del Observatorio Astronómico Centroamericano de Suyapa. Para formar con la cooperación internacional permanente, promociones de astrónomos que tendrán la responsabilidad de mover a Centro América más allá en campos relevantes de 
investigación científica. El conocimiento adquirido, uso y aplicaciones de instrumentación astronómica y tecnología espacial son de particular significado en el adelantamiento del objetivo general del OACS, y por supuesto, como una contribución para el desarrollo sostenible del país y la región. Por lo tanto, con el objeto de fortalecer este proyecto, la comunidad internacional de astrónomos puede ayudar proporcionando profesores visitantes deseosos de venir a Honduras a colaborar en la medida que sean necesitados; y con fondos para becas para estudiantes graduados para terminar sus estudios al más alto nivel, mientras garanticen y los estimulen a permanecer en sus propios países de Centro América.

- Los Cursos Regionales Centro Americanos (CURCAA) son excelentes oportunidades para ensamblar astrónomos de primera línea en el mundo como profesores de las universidades Centro Americanas y estudiantes interesados en el establecimiento de una tradición astronómica en Centro América. De alguna manera, estos eventos representan un capitulo regional de un esfuerzo mayor realizado por la serie de Talleres UN/ESA sobre Ciencia Espacial Básica. Porque todas las universidades nacionales de Centro América han acordado organizar este tipo de eventos en una base anual, con el objeto de continuar este esfuerzo y reforzar este tipo de actividad, ayudas especiales de organizaciones internacionales interesadas, instituciones, sociedades y similares serán útiles. De esta manera un mayor número de participantes de los diferentes países de Centro América estarán garantizadas. Hasta ahora, para todos los CURCAA, todas las universidades nacionales Centro Americanas y otras organizaciones locales, la IAU y algunas otras universidades extranjeras y organizaciones han patrocinado participantes.

- Aún más, después de haber hospedado uno y participado en la mayoría de los UN/ESA Workshop sobre Ciencia Espacial Básica, hemos aprendido de las múltiples ventajas de juntar científicos de diferentes regiones del mundo buscando alcanzar objetivos y metas precisas. También, después que nuestros proyectos han sido presentados en tal foro internacional, nuestras autoridades nacionales son hechas advertir de la importancia de los campos de la astronomía y la ciencia espacial básica para el desarrollo de capacidades autóctonas. Esto es especialmente cierto después de haber creado pequeños grupos en los países centroamericanos que están conjuntamente buscando mecanismos que les puedan permitir a ellos establecer la astronomía y astrofísica como campos profesionales con la permanente cooperación internacional en sus propios países.

\section{Referencias}

1. Borovicka, J., Pineda de Carías, M. C., et al. Meteoroids. Praga, República Checa. 1998.

2. Pineda de Carías, M. C. The Astronomical Observatory of Honduras: A Project of International Cooperation. En: W. Wamsteker et al. Developing Basic Space Science World-Wide. The Netherlands: Kluwer Académic Publisher; 2004. p.101-111.

3. Pineda de Carías, M. C. Astronomy for Developing Countries, IAU Special Session at the 24th General Assembly. ASP Conference Series. SPS, 69. Michigan. 2001. 
4. Pineda de Carías, M. C. Conference Proceedings. American Institute of Physics. New York: AIP; 1995. 320, 13.

5. Pineda de Carías, M. C. Mensaje al Futuro: 10 años de Astronomía Centroamericana en Honduras. OACS/UNAH. 2001.

6. United Nations General Assembly. Report on the Third UN/ESA Workshop on Basic Space Science, organized in cooperation with the GObernment of Nigeria and hosted by University of Nigeria, Nsukka, and the Obafemi Awolowo University, Ile-Ife, Lagos, Nigeria. 18-22 October 1993, Doccument A/AC.105/592/Add.1. Viena, Austria: 1993.

7. United Nations General Assembly. Report on the Seventh UN/ESA Workshop on Basic Space Science, 1998, Small Astronomical Telescopes and Satellites in Education and Research, hosted by the Observatorio Astronómico de la Universidad Nacional Autónoma de Honduras, on behalf of the Government of Honduras. Document A/AC.105/682; and COPAR Information Bulletin, 141, 9. Viena, Austria.

8. Wamsteker, W., Skillen, I., Ponz, J. D., de la Fuente, A., Barylak, M. and Yurrita, I. Astrophysics and Space Science. The Netherlands. 2000. 273 (1/4): 155-161.

9. Wamsteker, W. and Shustow B. M. The World Space Observatory/Ultraviolet (WSO/UV) Project. En: W. Wamsteker et al (ed). Developing Basic Space Science World-Wide. The Netherlands: Kluwer Académic Publisher; 2004. p.373-376. 